\title{
Uniformly Secondary Modules over Commutative Ring
}

\author{
Abdoljavad Taherizadeh; \\ Department of Mathematical Sciences and Computer \\ Kharazmi University, Tehran, Iran
}

Received: 5 Jun 2014

Revised: 1 July 2017

\begin{abstract}
In [1] the notion of "uniformly ideal" was introduced and developed the basic theory. In this article we introduce and develop a theory which, in a sense, dual to that this is, the notion of "uniformly secondary module".
\end{abstract}

Keywords: Uniformly secondary, Artinian modules.

Mathematics Subject Classification MSC(2000).13C05,13E10.

\section{Introduction}

Throughout this paper, all rings are commutative with non-zero identity and $\mathbb{N}$ (resp. $\mathbb{N}_{0}$ ) denotes the positive (resp-non-negative) integers.

Cox and Hetzel introduced and developed the basic theory of "uniformly primary ideals" for commutative rings [2]: They called a proper ideal Q of R uniformly primary if there exists a positive integer $n$ such that whenever $r, s \in R$ satisfy $r s \in Q, r \notin$ $\mathrm{Q}$, then $\mathrm{s}^{\mathrm{n}} \in \mathrm{Q}$. Also a uniformly primary ideal $\mathrm{Q}$ has order $\mathrm{k}$ and is written $\operatorname{ord}_{R} Q=$ $k$, or simply ord $\mathrm{Q}=\mathrm{k}$ if $\mathrm{k}$ is the smallest positive integer for which the aforementioned property holds. We introduce and develop a theory which is, in a sense, dual to uniformly primary notion, that is, uniformly secondary modules.

In section 2, we present the notion of uniformly secondary modules along with two other notions: "strongly secondary module" and "weakly secondary module". In addition, we provide two important characterizations of uniformly secondary modules (Proposition 2.10 and Theorem 2.11). Moreover, we get a connection between uniformly secondary and strongly secondary, while by some examples (Examples 2.4 and 2.9) we demonstrate that there is no equivalence, in general, between these 3 notions. 
Section 3 is devoted to developing some basic properties of uniformly secondary modules. Finally in section 4, we focus on uniformly modules in which the radical of their annihilator are finitely generated along with Artinian secondary modules. In particular, proposition (4.1) shows that whenever the radical of the annihilator of a secondary module is finitely generated, or instead, the secondary module is Artinian, then it is uniformly secondary. As a consequence of these results, we conclude (in corollary 4.2) that if $\mathrm{N}$ is a secondary R-module, then the Noetherianess of $\mathrm{R}$ or Artinianess of $\mathrm{N}$ guarantees that $\mathrm{N}$ is strongly secondary and hence uniformly and weakly secondary.

We recall from [4] that the non-zero $\mathrm{R}$-module $\mathrm{N}$ is called a secondary module whenever for each $r \in R$, the homothety $\mathrm{N} \stackrel{\mathrm{r}}{\rightarrow} \mathrm{N}$ is surjective or there exists $\mathrm{n} \in \mathrm{N}$ such that $r^{n} \mathrm{~N}=0$. If $\mathrm{N}$ is a secondary $\mathrm{R}$-module, then $\mathrm{p}:=\sqrt{0:{ }_{\mathrm{R}} \mathrm{N}}$ is a prime ideal and $\mathrm{N}$ is called a p-secondary R-module. For more results about secondary modules see [4].

\section{Characterization of uniformly secondary modules}

We start this section with some basic definitions.

Definition2.1. An R-module $M \neq 0$ is said to be uniformly secondary whenever there exists a positive integer $n$ such that for all $r \in R$ if the mapping $M \rightarrow M$ is not subjective, then $\mathrm{r}^{\mathrm{n}} \mathrm{M}=0$. If $\mathrm{N}$ is a uniformly secondary R-module, then the co- order of $\mathrm{N}$ is the least positive integer $\mathrm{n}$ such that the above-mentioned property holds. If $\mathrm{N}$ has co-order $\mathrm{k}$, we write co $-\operatorname{ord}_{\mathrm{R}} \mathrm{N}=\mathrm{k}$ (or simply,co-ord $\mathrm{N}=\mathrm{k}$ ). For example $\mathrm{Z}_{2}$ nis a uniformly secondary Z-module with co $-\operatorname{ord}_{\mathrm{z}} \mathrm{Z}_{2^{n}}=\mathrm{n}(\mathrm{n} \in \mathbb{N})$.

Definition2.2. Let $p \in \operatorname{Spec}(\mathrm{R})$. Then

An R-module $\mathrm{N}$ is said to be strongly secondary if $\mathrm{N}$ is $\mathrm{p}$ secondary and $\mathrm{p}^{\mathrm{t}} \mathrm{N}=0$ for some $\mathrm{t} \in \mathrm{N}$. If $\mathrm{N}$ is an strongly secondary modules, then the least positive integer e for which $\mathrm{p}^{\mathrm{e}} \mathrm{N}=0$ is called the co-exponent of $\mathrm{N}$ and we write co$\exp _{\mathrm{R}}(\mathrm{N})=$ e or simply co $-\exp (\mathrm{N})=$ e.

We call an R-module $\mathrm{N}$ weakly secondary if $\mathrm{N}$ is p-secondary and there is $\mathrm{r} \in \mathrm{R}$ such that $\mathrm{rN} \neq 0$ and $\mathrm{p}(\mathrm{rN})=0$.

Remark2.3. (i) It is easy to see that every strongly secondary modules is a weakly secondary module, but the converse is not, in general, true, as the next example (2.4) shows. 
(ii)If $\mathrm{R}$ is a Noetherian ring, then every secondary R-module is strongly secondary and it follows from (i) and next proposition that the above three concepts are the same. We recall that the next example is a modification of an example given in [2, page 2].

Example2.4. Let $\mathrm{K}$ be a field and let $\mathrm{R}:=\mathrm{K}\left[\mathrm{x}_{1}, \mathrm{x}_{2}, \ldots.\right]$ and $\mathrm{M}:=\frac{\mathrm{R}}{\mathrm{q}}$ where $\mathrm{q}:=$ $\left(\left\{x_{i}^{2}\right\}_{i=1}^{\infty},\left\{x_{1} x_{i}\right\}_{i=2}^{\infty}\right) R$. Then $p:=\left(x_{1}, x_{2}, \ldots, x_{n} \ldots\right) R$ is a maximal ideal of $R$ and $\sqrt{\left(0_{R}: M\right)}=p$. Thus $M$ is $p$-secondary, $x_{1} M=0$ and $p\left(x_{1} M\right)=0$ and so $M$ is a weakly secondary module. But for each positive integer $n, x_{1} x_{2} \ldots x_{n+1} \in p^{n}$ and $\mathrm{x}_{2} \mathrm{x}_{3} \ldots \mathrm{x}_{\mathrm{n}+1} \mathrm{M} \neq 0$. Thus $\mathrm{M}$ is not a strongly seconday module.

Now, we consider the relation between uniformly secondary and strongly secondary modules.

Propostion2.5. Let $M$ be a strongly $p$ secondary $R$-module. Then $M$ is uniformly secondary. Further, co - ord $M \leq$ co $-\exp M$

Proof. Let $M$ be a strongly secondary module. Then there is $t \in N$ such that $p^{t} M=$ 0 . suppose that $\mathrm{M} \stackrel{\mathrm{r}}{\rightarrow} \mathrm{M}$ is not surjective, then $\mathrm{r} \in \mathrm{p}$ and so $\mathrm{r}^{\mathrm{t}} \mathrm{M}=0$

Corollary2.6. Let $p$ be prime ideal of $R$ and $M$ an R-module. Suppose that for some positive integer $n, \quad\left(0:_{M} p^{n}\right)\left(\operatorname{resp}, \frac{M}{p^{n} M}\right)$ is p-secondary (and hence strongly secondary). Then $\left(0:{ }_{M} p^{n}\right)\left(\right.$ resp,$\left.\frac{M}{p^{n} M}\right)$ is a uniformly secondary $R$-module of co-order at most $n$. In particular if $p$ is a maximal ideal, then for each positive $n$, $\left(0:{ }_{M} p^{n}\right)\left(\right.$ resp,$\left.\frac{M}{p^{n} M}\right)$ if is not zero, is a uniformly secondary module of co-order at most $n$.

In general, the co-order of the secondary submodules $\left(0:{ }_{M} p^{n}\right)\left(\right.$ resp, $\left.\frac{M}{p^{n} M}\right)$ may be smaller than $\mathrm{n}$. For example let $\mathrm{R}$ be a valuation ring with maximal ideal $\mathrm{m}$ which is not principal and $\mathrm{M}$ an R-module. Then since $\mathrm{m}^{2}=\mathrm{m}$. for each $\mathrm{n} \epsilon \mathrm{N}$.

$$
\left(0:_{M} m^{n}\right)=\left(0:{ }_{M} m\right)\left(\operatorname{resp} \cdot \frac{M}{m^{n} M}=\frac{M}{m M}\right)
$$

is a uniformly secondary module of co-order 1.

The next corollary provides exmples of uniformly secondary modules of the form $\frac{\mathrm{M}}{\mathrm{p}^{\mathrm{n}} \mathrm{M}}$ of co-order exactly $\mathrm{n}$.

Corollary2.7. Let $\mathrm{M}:=\mathrm{Z}$. Then $\frac{\mathrm{Z}}{2^{\mathrm{n}} \mathrm{Z}}$ is a uniformly secondary module of co-order exact $\mathrm{n}(\mathrm{n} \in \mathbb{N})$

Exmple2.8. below shows that there is a strongly secondary R-module $\mathrm{N}$ with co-order $\mathrm{N}<\operatorname{co}-\exp \mathrm{N}$. 
Exmple2.9 shows that there is a uniformly secondary module which is not a weakly secondary module, and so there is a uniformly secondary module which is not a strongly secondary module. Examples 2.8 and 2.9 are respectively modifications of Exmple 6 and 7 of [2].

Example2.8. Let $R=Z_{2}\left[x_{1} \ldots x_{n}\right]$ where $n \geq 2$ and let $\mathrm{q}:=\left(x_{1}^{2}, \ldots, x_{n}^{2}\right) R$. Then $\frac{R}{q}$ is a $p:=\left(x_{1} \ldots x_{n}\right) \mathrm{R}$ - secondary R-module such that $p^{n+1} \subseteq q$. Thus $P^{n+1}\left(\frac{R}{q}\right)=0$. But $x_{1} x_{2} \ldots x_{n} \in p^{n} \backslash q$ and so $p^{n}\left(\frac{R}{q}\right) \not \equiv 0$. So $\frac{R}{q}$ is a strongly p-secondary module with co-exp $\frac{R}{q}=\mathrm{n}+1$. For each $f \in p,\left(\frac{R}{q}\right) \neq \frac{R}{q}$ and $f^{2}\left(\frac{R}{q}\right)=0$. Thus co - ord $\left(\frac{R}{q}\right)=2$.

Example2.9. Let $R=Z_{2}\left[x_{1} \ldots x_{n}\right]$ and $q:=\left(\left\{x_{i}^{2}\right\} ; i \geq 1\right)$ Then $\frac{R}{q}$ is an $p:=\left(x_{1} \ldots x_{n}, \ldots\right) R$ -secondary R-module. For each $f \in p, f^{2}\left(\frac{R}{q}\right)=0$. so $\frac{R}{q}$ is a uniformly p - secondary R- module with co - ord $\left(\frac{R}{q}\right)=2$, but for any distinct positive integers $i_{1}, i_{2}, \ldots i_{n}$, $x_{1}, x_{2}, \ldots x_{n} \notin q$. it follows that there is no $f \in R \backslash q$ with $f p \subseteq q$. Thus $\frac{R}{q}$ Is not a weakly secondary module and so is not a strongly secondary module

The following proposition provides a useful characterization of uniformly secondary modules.

Proposition2.10. The $R$-module $N$ is a uniformly p-secondary module if and only if the following two conditions hold.

i) $N$ is a p-secondary module

ii) There is a positive integer $n$ such that $p=\left\{r \in R r^{n} \in\left(0:_{R} N\right)\right\}$.

In addition co- $\operatorname{ord}_{R} N=k$ if and only if $k$ is the least integer for which condition (ii) holds

Proof $\Rightarrow$ ) Is trivial.

$\Leftrightarrow$ Suppose that $\mathrm{N}$ is an $\mathrm{R}$-module which satisfies (i) and (ii).Let $r \in R$ be such that the mapping $\mathrm{N} \stackrel{r}{\rightarrow} \mathrm{N}$ is not subjective. Then by (i) $\mathrm{r} \in \mathrm{p}$ and $\operatorname{so}^{\mathrm{n}} \mathrm{N}=0$. Thus $\mathrm{N}$ is a uniformly secondary R-module.

The last statement follows from definition of co-order and (i) and (ii) above.

Next we are going to provide a content where the concept of 'uniformly secondary modules' and 'strongly secondary module' are equivalent

In next theorem and its corollary and 2.12, Q denotes the set of rational numbers. 
Theorem2.11. Let $R$ be a $Q$-algebra and $N$ an $R$-module. Then $N$ is a uniformly secondary module of co-order $k$ if and only if $N$ is a strongly secondary module of coexponent $k$.

Proof. Suppose that $\mathrm{N}$ is a uniformly $\mathrm{p}$-secondary module of co-order $\mathrm{k}$. if $\mathrm{k}=1$, the result is trivial. So suppose that $k \geq 2$. By proposition (2.10) there is $r \in p$ such that $r^{k} N=$ 0 , but $\mathrm{r}^{\mathrm{k}-1} \mathrm{~N} \neq 0$. So $\mathrm{p}^{\mathrm{k}-1} \mathrm{~N} \neq 0$. We show that $\mathrm{p}^{\mathrm{k}} \mathrm{N}=0$. Let $\mathrm{a}_{1}, \mathrm{a}_{2}, \ldots, \mathrm{a}_{\mathrm{k}} \in \mathrm{p}$. since $\mathrm{k}$ is a $\mathrm{Q}-$ algebra, by [2; lemma 9] the product $\mathrm{a}_{1} \mathrm{a}_{2} \ldots \mathrm{a}_{\mathrm{k}}$ can be expressed as a sum of a unit of $\mathrm{R}$ times a sum of k-th powers of element of p. Again by proposition $(2.10)\left(a_{1}, \ldots a_{k}\right) N=0$, and so $\mathrm{p}^{\mathrm{k}} \mathrm{N}=0$.

Conversely, suppose $\mathrm{N}$ is a strongly p-secondary R-module with co-exp. k. By proposition $2.5 \mathrm{~N}$ is a uniformly p-secondary module with co-ord $\mathrm{R}_{\mathrm{N}} \mathrm{N}$. By the same argument as 'only if' statement the co-order of $\mathrm{N}$ can not be strictly less than $\mathrm{k}$, otherwise, $\mathrm{p}^{\mathrm{k}-1} \mathrm{~N}=0$, a contradiction to the fact that the co-exponent of $\mathrm{N}$ is $\mathrm{k}$. thus coord $\mathrm{N}=\mathrm{k}$.

Corollary2.12. Let $R$ be a $Q$-algebra and $N$ be a p-secondary $R$-module. Then $N$ is a uniformly p-secondary module if and only $\frac{P}{\left(0:_{R} N\right)}$ is a nilpotent ideal of $\frac{P}{\left(0:_{R} N\right)}$

The next example shows the existence of a weakly secondary module which is not a uniformly secondary module.

Example2.13. With the same assumptions and notations as Example (2.4), let R be a Qalgebra. By that example $\frac{R}{q}$ is a weakly secondary module which is not strongly secondary module and so by Theorem (2.11) is not a uniformly secondary module.

For the next proposition we recall two definition from [2].

Definition2.14. Let $q$ be a p-primary ideal of $\mathrm{R}$ :

(i) The ideal $\mathrm{q}$ is called a Noether primary ideal if $\mathrm{p}^{\mathrm{n}} \subseteq \mathrm{q}$ for some $\mathrm{n} \in \mathbb{N}$. if $\mathrm{q}$ is a Neother strongly primary ideal, then following [1], the smallest positive integer $n$ for which $\mathrm{p}^{\mathrm{n}} \subseteq \mathrm{q}$, is called the exponent of $\mathrm{q}$ and is written as $\mathrm{e}_{\mathrm{R}}(\mathrm{q})=\mathrm{n}$, or simply, $\mathrm{e}(\mathrm{q})=\mathrm{n}$.

(ii) The ideal $q$ is called a Mori strongly primary ideal if there exists $r \in R / q$ such that $\mathrm{rp} \subseteq \mathrm{q}$. see [5]

Proposition2.15. Let $N$ be a p-secondary R-module. Then:

(i) If $N$ is uniformly p-secondary module of co-order $k$, then $0_{: R} N$ is a uniformly $p$ primary ideal of order $k$. 
(ii) If $N$ is a strongly p-secondary module of co-expenent e, then $0_{: R} N$ is a Noether strongly p-primary ideal of exponent $e$.

(iii) If $N$ is a weakly p-secondary module, the $O_{: R} N$ is a Mori strongly p-primary ideal of $R$.

Proof. By $[4 ;(1.2)]$ if $\mathrm{N}$ is a p-secondary R-module, then $0: \mathrm{R}$ is a p-primary ideal. To prove (i), let co-ord $N=k$ and $r, s \in R$ be such that $r s \in\left(0_{: R} N\right)$ and $r \notin\left(0_{: R} N\right)$. It follows that $\mathrm{sN} \neq \mathrm{N}$ and so by the definition of uniformly secondary and co-order, we deduce that $s^{k} \in\left(0_{: R} N\right)$. Now the last part of (i) follows easily from definition of order and co-order.

(i), (iii). The proof is straightforward.

\section{Some properties of uniformly secondary modules}

In this section we prove some properties of uniformly secondary modules. The first one compare the co-orders of these modules.

Proposition3.1. Let $N_{1} \subseteq N_{2}$ be two uniformly p-secondary submodules of an R-module. Then co-ord $N_{1} \leq$ co-ord $N_{2}$. Also if $N_{2}$ is a quotient of $N_{1}$, then co-ord $N_{2} \leq$ co-ord $N_{1}$ Proof. Let $N_{1} \subseteq N_{2}$ and $\mathrm{n}:=$ co-ord $N_{1}, \mathrm{~m}:=$ co-ord $N_{2}$. If $N_{2} \stackrel{r}{\rightarrow} N_{2}(\mathrm{r} \in \mathrm{R})$ is not surjective, then $\mathrm{r}^{\mathrm{m}} N_{2}=0$ and so $\mathrm{r}^{\mathrm{m}} \mathrm{N}_{1}=0$. Thus $\mathrm{n} \leq \mathrm{m}$. the proof of the last assertion is similar.

Remark3.2. It is possible that uniformly p-secondary submodules $N_{1}$ and $N_{2}$ of an $R$ module be such that $\mathrm{N}_{1} \subseteq \mathrm{N}_{2}$ but co-ord $\mathrm{N}_{1}=$ co-ord $\mathrm{N}_{2}$. for example $\mathrm{Z}_{4} \oplus \mathrm{Z}_{2}$ and $\mathrm{Z}_{4}$ are 2z-uniformly $\mathrm{Z}$-modules (and we can consider $\mathrm{Z}_{4}$ as an $\mathrm{Z}$-submodule of $\mathrm{Z}_{4} \oplus \mathrm{Z}_{3}$ ) and coord $\mathrm{Z}_{4} \oplus \mathrm{Z}_{2}=$ co-ord $\mathrm{Z}_{4}=2$.

Proposition3.3. let $\left\{N_{i}\right\}_{i \in I}$ be a collection of uniformly p-secondary submodules of an $R$ module $M$ and co-ord $N_{i}=k$, for all $i \in I$ where $k \in N$. Then $\Sigma_{i \in I} N_{i}$ is a uniformly $p$ secondary submodule of $M$ of co-order $k$.

Proof. Put $\mathrm{N}:=\sum_{\mathrm{i} \in \mathrm{I}} \mathrm{N}_{\mathrm{i} \text {. }}$ then $\sqrt{0:_{\mathrm{R}} \mathrm{N}}=\sqrt{0:_{\mathrm{R}} \sum_{\mathrm{i} \in \mathrm{I}} \mathrm{N}_{\mathrm{i}}}=\mathrm{p}$ and $\mathrm{p}=\left\{\mathrm{r} \in \mathrm{R} \mid \mathrm{r}^{\mathrm{k}} \in(0: \mathrm{N})\right\}$. Next let $N \stackrel{r}{\rightarrow} N$ be such that $r N \neq N j$. Than there is $j \in I$ such that $r N_{j} \neq N_{j}$. Thus $r \in p$ and $\mathrm{r}^{\mathrm{k}} \mathrm{N}=0$, hence $\mathrm{N}$ is a uniformly p-secondary module of co-order at most $\mathrm{k}$. but co-ord $\mathrm{N}_{\mathrm{j}}=\mathrm{k}$ for all $\mathrm{j} \in \mathrm{I}$, and by Proposition 2.10 for a fixed $\mathrm{j} \in \mathrm{p}, \mathrm{k}$ is the least integer for which $=\left\{r \in R \mid r^{n} \in\left(0:{ }_{R} N_{j}\right)\right\}$. Thus there is $r \in p$ such that $r^{k-1} N_{j} \neq 0$, and so $r^{k-1} N \neq 0$. Hence co-ord $\mathrm{N}=\mathrm{k}$. 
In the next proposition we consider the behaviour of uniformly secondary modules under change of rings

Proposition3.4. let $\varphi$ : $A \rightarrow B$ be a ring homomorphism and $N$ be a uniformly $q$-secondary submodule of a B-module $M$. then $N$ is a uniformly $\varphi^{-1}(q)$-secondary submodule of $M$ when we consider $M$ as an A-module via $\varphi$ and moreover co-ord ${ }_{A} N \leq c o-\operatorname{ord}_{B} N$.

Remark3.5. The inequality between co-orders in Proposition (3.3) may be strict as the next example shows

Example3.6. let $R=K\left[x^{n}\right](n>1)$ and $S=K[x]$ where $K$ is a field and $\varphi$ in proposition 3.4 is the inclusion map. Then $N:=\frac{K[x]}{\left.<x^{n}\right\rangle}$ is a uniomly secondary S-module with co-ord ${ }_{\mathrm{S}} N=$ n. but $N$ is a uniformly secondary R-module with $\operatorname{co-} \operatorname{ord}_{\mathrm{R}} N=1$.

Corollary3.7. let $N$ be an $R$-module with $I \subseteq$ AnnN. Then $N$ is a uniformly p-secondary $R$-module of co-order $k$ if and only if $N$ is a uniformly $\frac{P}{I}-$ secondary $\frac{R}{I}-$ module of co-order $k$.

Proof. Suppose that $\mathrm{N}$ is a uniformly $\frac{\mathrm{P}}{\mathrm{I}}-$ secondary $\frac{\mathrm{R}}{\mathrm{I}}-$ module of co-order $\mathrm{k}$.

By Proposition (3.9) $\mathrm{N}$ is a uniformly p-secondary R-module of co-ord $\mathrm{R}_{\mathrm{N}} \leq \mathrm{k}$. By proposition (2.9) there is $r+I \in \frac{P}{I}$ such that $(r+I)^{k-1} N \neq 0$. Thus $r^{k-1} N \neq 0$ and so again by Proposition (2.9) co-ord $\mathrm{k}=\mathrm{k}$.

Conversely, suppose that $\mathrm{N}$ is a uniformly p-secondary R-module with co-ord $\mathrm{R} N=\mathrm{k}$. then it is easy to see that $\mathrm{N}$ is a uniformly $\frac{\mathrm{P}}{\mathrm{I}}-$ secondary $\frac{\mathrm{R}}{\mathrm{I}}-$ module with co $\operatorname{ord}_{\frac{R}{I}} \mathrm{~N} \leq \mathrm{k}$. but by Proposition (3.3) k $=$ co-ord $_{\mathrm{R}} \mathrm{N} \leq$ co $-\operatorname{ord}_{\frac{\mathrm{R}}{\mathrm{I}}} \mathrm{N}$ and so co-ord $\mathrm{R}_{\mathrm{R}} \mathrm{N}$ $=\operatorname{co}-\operatorname{ord}_{\frac{\mathrm{R}}{\mathrm{I}}} \mathrm{N}=\mathrm{k}$.

Proposition3.8. let $S$ be a multiplicatively closed subset of $R$ and $N$ be a uniformly $p$ secondary $R$-module. Then $S^{-1} N=0$ or $S^{-1} N$ is a uniformly $S^{-1} p$-secondary $S^{-1} R$-module and co-ord ${ }_{S-1 R} S^{-1} N \leq$ co-ord $_{R} N$.

Proof. The proof is straightforward.

\section{Uniformly secondary modules over Noetherian rings and Artinian uniformly secondary modules}

In this section we examine the properties of secondary modules over Noetherian rings and Artinian secondary modules as well, and we see that in these situations, the notions 
'uniformly secondary', 'strongly secondary', weakly secondary and secondary are equivalent

Proposition4.1. let $N$ be a secondary $R$-module. Then $N$ is a strongly secondary module and so a uniformly secondary module, if one of (i) or (ii) below holds:

(i) $p$ is a finitely generated ideal

(ii) $N$ is an Artinian-module.

Proof. (i). First we suppose that $\mathrm{p}$ is a finitely generated ideal of $\mathrm{R}$, with a generating set for example $r_{1}, \ldots, r_{k}$. Then there is positive integers $t_{1}, \ldots, t_{k}$ such that $r_{i}^{t_{i}} N=0$. Put $\mathrm{n}:=\sum_{\mathrm{i}=1}^{\mathrm{k}}\left(\mathrm{t}_{\mathrm{i}}-1\right)+1$. Then $\mathrm{p}^{\mathrm{n}} \mathrm{N}=0$, and so $\mathrm{N}$ is a strongly secondary module and by Propositon 2.4 a uniformly secondary module

(ii) let $N$ be an Artinian module. By [3, lemma 3] there is a $\subseteq$ p such that a is finitely generated and $\left(0:{ }_{N} a^{t}\right)=\left(0:{ }_{N} p^{t}\right)$ for all $t \in \mathbb{N}$. by (i) there is $n \in N$ such that $a^{n} N=0$ and so $\mathrm{N}=\left(0:{ }_{N} a^{n}\right)=\left(0:{ }_{N} p^{n}\right)$. Hence $p^{n} N=0$, and so $N$ is a strongly secondary modules and hence a uniformly secondary module by Propostion 2.9.

Corollary4.2. let $N$ be an R-module. Suppose that $\mathrm{R}$ is a Noetherian ring (resp. $N$ is an Artinian module). Then the following are equivalent:

1) $N$ is a secondary module;

2) $N$ is a uniformly secondary module;

3) $N$ is a strongly secondary module;

4) $N$ is a weakly secondary module.

\section{References}

1. Cox J-A., Hetzel A. J., "Uniformly primary ideals", J. pure Appl. Algebra 212 (2008) 1-8.

2. Bourbaki N., "Commutative algebra, Springer-verlag", Berlin (1989) (Chapters 1-7).

3. Kirby D., "Artinian modules and Hilbert polynomials", Quart, J. Math. Oxford (2) 24 (1973) 47-57.

4. Macdonald I.G., "Secondary representation of modules over a commutative ring", symposia Matematica 11 (1973) 23-43.

5. Mori S., "Über die symmetrie des pradikates "Relative prime"", II, I, Sci. Hiroshima Univ. Ser. A 15 (1971) 79-85. 